\title{
COMPLICATIONS OF LOCALIZED PULMONARY EMPHYSEMA IN CHILDHOOD
}

\author{
BY \\ S. van CREVELD, M.D., and J. van den BROECKE, M.B. \\ (From the Children's Clinic of the Municipal University of Amsterdam, Holland)
}

Our knowledge of the causes of interstitial pulmonary emphysema and its eventual complications has been greatly extended by clinical and experimental observations made principally during the last ten years. We can now assume with confidence that interstitial pulmonary emphysema is usually caused by rupture or leakage of the alveoli, due to trauma or raised intra-pulmonary pressure, or alternatively is due to spontaneous rupture of alveoli. Air which enters the interstitial tissues from the alveoli is able to shift position, and to pass along the sheaths of the blood vessels. Macklin (1937, 1939) and Fischer and Macklin (1940) have shown experimentally that the entrance of air into the sheath-system is effected by way of multiple minute tears in the base of the alveoli, which are closely connected with the finer ramifications of the pulmonary vessels. The cause of these tears is usually a rise of intra-alveolar pressure, occurring as a result of transient bronchial obstruction of the check-valse type; this we now know may sometimes lead to localized pulmonary emphysema and ultimately to cavitation of the lungs. It is, therefore, not surprising that localized bullous emphysema and interstitial pulmonary emphysema (occurring apart from birth trauma or asphyxia) have often been observed in pneumonia, pertussis, stenosis of the larynx, and asthma.

Attention has been drawn to this cavity-formation resulting from localized bullous emphysema in pneumonia in childhood by Tillema (1940) of this clinic, and by a number of other authors (Caffey, 1940; Wissler, 1938; Benjamin and Childe, 1939; and more recently by Leopold and Kratzman, 1945). In the above-mentioned conditions, thick and sticky sputum readily leads to obstruction of the trachea, bronchi, and bronchioli, whilst coughing further increases the intra-pulmonary pressure. Macklin has also shown that distension of the alveolar walls can exist for a long time without rupture occurring. When, however, interstitial pulmonary emphysema has been established, air can then pass (principally along the sheaths of the blood vessels) to the mediastinum, resulting in mediastinal emphysema. Increased pressure on the pulmonary vessels will at the same time affect the circulation to a greater or less extent. In cases of post-operative atelectasis, associated with forcible coughing to clear the obstructed bronchus, pneumo-mediastinum has occurred, owing to rupture of the mediastinal pleura.

In the mediastinum, the air can be gradually resorbed, or may lead to further obstruction of the circulation from pressure on the heart and great vessels. When the pressure of air increases to a great extent, however, the air will seek an outlet from the mediastinum; this has repeatedly been observed in animal experiments when the lungs were forcibly inflated. The easiest exit of air from the mediastinum appears to be by way of the connective tissue around the blood-vessels, and may occur in one of three different directions: (1) to the subcutaneous and deeper tissues of the neck (sometimes also of the shoulders, face, trunk and extremities), (2) to the pleural cavity, and (3) along the aorta and oesophagus, through the diaphragm to the retroperitoneal tissues of the flanks and perirenal areas.

Subcutaneous emphysema in the neck, however, is not necessarily due to air in the mediastinum, and before the latter condition can be assumed, other possible causes such as wounds of the thorax communicating with the lung, or air originating from the mouth or nasal cavity, must be excluded.

Escape of air from the mediastinum to the pleural cavity causes a pneumothorax, and bullous emphysema can, therefore, lead to a pneumothorax by way of interstitial and mediastinal emphysema (cf. Hamman, 1945). Bullous emphysema may also give rise to a pneumothorax, however, by the bursting of a bulla situated on the surface of the lung. This took place, for example, in the patient with infectious reticulo-endotheliosis described by van Creveld and ter Poorten (1935), where a great many such bullae were present on the surface of the lungs. At autopsy, the opening in the ruptured bulla which had caused the pneumothorax could be identified immediately.

That air from the mediastinum can find its way into the pleural cavity has clearly been demonstrated experimentally; the forcible introduction of air into the lungs of an animal results in a pneumothorax. When sudden death occurs in such an experiment, bilateral pneumothorax with collapse of both lungs is found at autopsy. However, under these circumstances, there is no trace of a rupture or perforation of the visceral pleura. In Hamman's opinion (confirmed by a recent observation of our own), tension pneumothorax may be secondary to 
pneumo-mediastinum rather than to a valvular rupture through the pleura which allows air to enter but not to leave the pleural cavity.

The severity of the symptoms will depend on the volume of air which enters and remains in the mediastinum after the occurrence of interstitial emphysema. When mediastinal emphysema is followed by extensive subcutaneous emphysema in the neck, the symptoms directly due to mediastinal emphysema are usually few and relatively insignificant. Thus subcutaneous emphysema in the neck may actually be the first symptom of mediastinal and interstitial emphysema respectively.

Although the causes of interstitial emphysema with its sequelae, and those of localized bullous emphysema, are usually the same, it is exceptional for both types of emphysema to be present simultaneously. That this is possible, however, and also that symptoms of localized bullous emphysema can exist at the same time as the sequelae of interstitial emphysema (e.g. emphysema of the neck) is proved by one of the cases discussed below.

The severe symptoms which arise when much air is present in the mediastinum closely resemble those caused by increased intra-pericardial pressure. Dyspnoea and cyanosis may appear early, due to pressure on blood-vessels; these symptoms gradually become more severe, and unless relieved are likely to prove fatal. In Macklin's experiments, it was found that when air was blown into a lobe of the lung by means of a catheter, there appeared around the tip of the catheter a balloon-shaped emphysematous portion of lung surrounded by a layer of atelectasis. Around the vessels descending to the mediastinum, Macklin observed numerous air-bubbles, forming a canal in the sheath of the vessel. When emphysema was produced in animals by the introduction of air directly into the mediastinum, and death followed, it was found that the heart and great vessels in the thorax were surrounded by air-bubbles.

The presence of air in the mediastinum can sometimes be seen very clearly in the $x$ ray, whilst in other cases it must be looked for more carefully. In the antero-posterior view, a sharply demarcated transparent band can sometimes be observed in the line of the pericardium. Hamman considers that this localization of mediastinal emphysema is sometimes wrongly regarded as a pneumo-pericardium; as a rule, according to this author, it is in fact a 'pneumo-praecordium.' Others, however, regard it as a true pneumo-pericardium. Such an appearance has been observed in cases of whoopingcough, pulmonary tuberculosis, broncho-pneumonia, stenosis of the larynx, after severe physical exertion, after operations on the neck, thorax and abdomen, following traumatic perforations of the oesophagus, in infections with gas-producing organisms, and after necrosis due to radium (Graebner, 1939). In view of Macklin's experiments and of the case discussed below, we believe that in these circumstances it is quite possible for mediastinal emphysema to produce a pneumo-pericardium, the air reaching the pericardium along the sheaths of the blood- and lymph-vessels (compare the passage of dust along the same route).

When interstitial emphysema occurs as a result of excessive pressure in the alveoli, such factors as weakness of the alveolar walls due to infectious, toxic or hereditary influences, are likely to play a part in its production. Predisposed individuals may suffer from repeated attacks of interstitial emphysema, and this is also true of spontaneous pneumothorax.

\section{Case reports}

Case 1. Baby J., aged ten weeks, was admitted in August, 1945, to the Children's Clinic for feeding disorder. The parents were healthy and the family history essentially negative. The infant had been born at full term, weighing 3,500 g., and was not asphyxiated. Apart from the feeding difficulty at the time of admission, the infant had been healthy.

ON ADMISsion. The child appeared to be moderately ill; its nutritional state was poor, and its weight $2,700 \mathrm{~g}$. The heart and lungs showed no abnormalities. Slight albuminuria was present. The blood picture was normal.

Progress. The digestive disturbance soon improved, but recurrent abscesses of the skin of varying size appeared. The Pirquet tuberculin test was negative. On November 5, 1945, the infant developed rhinitis, and the following day began to cough. On the night of November 6-7, the infant had two attacks of extreme dyspnoea, in which it became deeply cyanotic and perspired freely; the pulse was slow, and there was inspiratory retraction of the jugular region and lower part of the thorax. The temperature was not affected, and there were no convulsions.

On November 7 , the infant had several attacks of vomiting and coughing, and during the night a further attack of dyspnoea and cyanosis. No abnormal physical signs were detected in the heart and lungs. The following day the child's condition had deteriorated. Examination of the left flank and left upper chest posteriorly showed some impairment of percussion note and diminished breath sounds. The heart was not displaced, and no murmur was heard. The heart sounds were loud. The blood picture showed 24,600 white blood cells per c.mm., of which 64 per cent. were polymorphonuclear, 33 per cent. lymphocytes, and 3 per cent. monocytes; toxic granulation was marked. The same afternoon, shortly after a feed, a further attack of severe dyspnoea and cyanosis occurred, lasting about two minutes; the infant was ashen-coloured during the attack, and this colour persisted for some considerable time. The respiration remained shallow, the alae nasi working, and severe inspiratory retractions continued. The pulse rate was 106 per minute and the temperature $35 \cdot 8^{\circ} \mathrm{C}$. In the oxygen tent the child became quieter and had no further attacks, though some dyspnoea remained.

On November 9 there was impaired percussion note and reduction of breath sounds over the left upper and right lower lobes and in the left flank. $X$ ray of the chest (fig. 1) showed a sharply demarcated, rather wide transparent area along the whole of the left border and right outer border of the heart. Translucency of this type can only represent a pneumo-pericardium. There was also an 


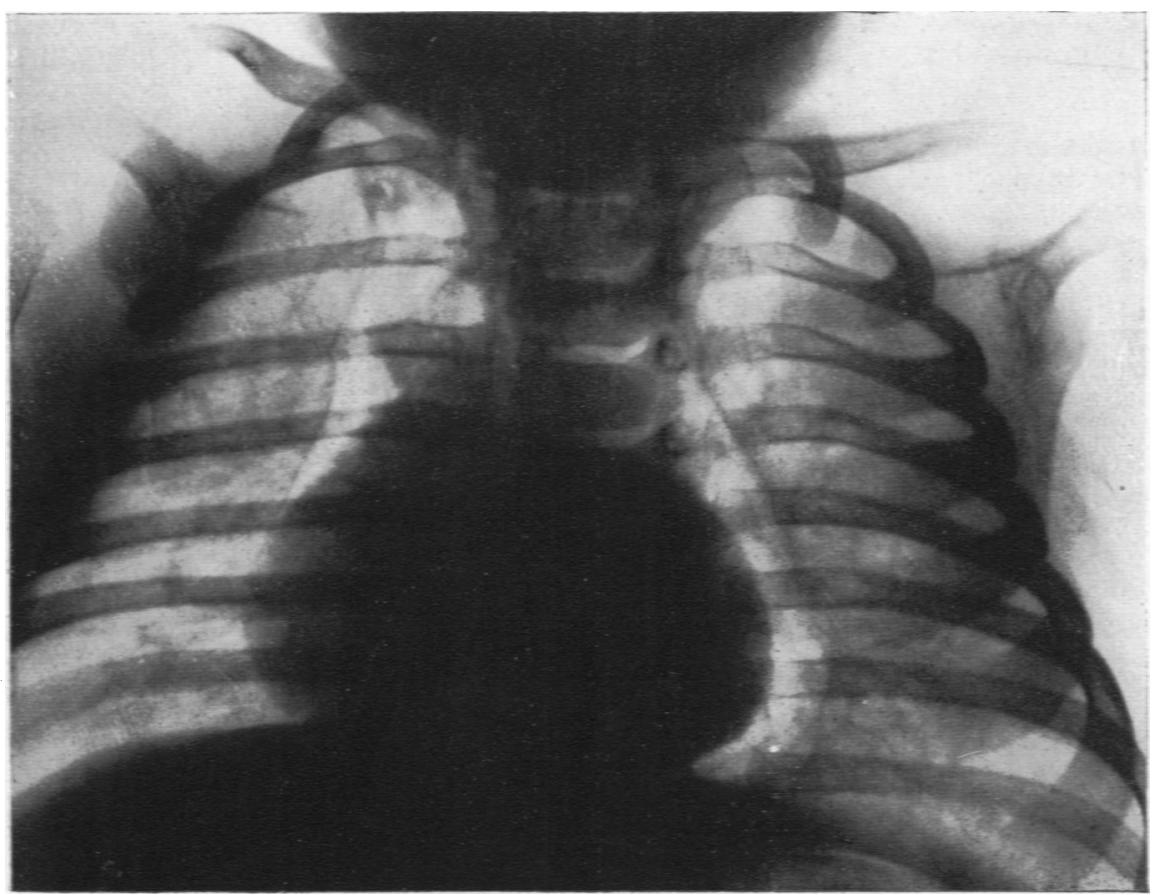

FIG. 1-X ray of chest, Case 1, showing a sharply demarcated, rather wide transparent area along the whole of the left border and right outer border of the heart.

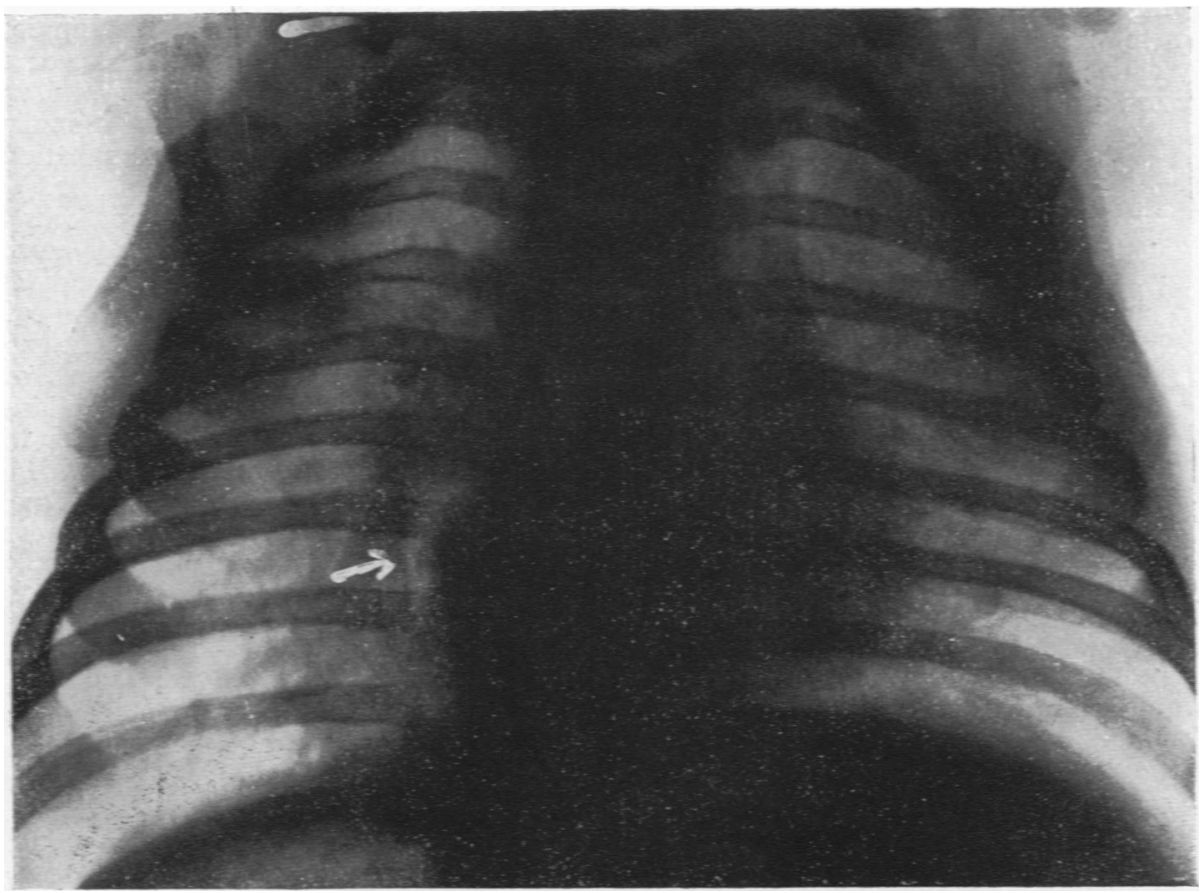

Fig. 2.- $X$ ray of the chest, Case 1, taken four days after $x$ ray in fig. 1, and still showing some air adjacent to the right border of the heart. 
accumulation of air in the left and right sides of the mediastinum. A second $x$ ray (fig. 2) taken four days after the first, still showed some air adjacent to the right border of the heart, but a third $x$ ray, taken two weeks after the first, showed that this air had by then disappeared.

The clinical condition of the child improved rapidly, but some degree of dyspnoea remained for a considerable time.

Comment. Radiographic evidence was found of a pneumo-pericardium with pneumo-mediastinum in a child with broncho-pneumonia, who suffered from severe attacks of dyspnoea with cyanosis. Of the signs described in analogous cases, one was lacking, viz. the characteristic praecordial crepitation resembling the crackling of cellophane (Hamman's sign). It is obvious that in an infant of this age the subjective symptoms, e.g. pain caused by the pressure of air on the ramifications of the pulmonary artery, or the anginal pain due to pressure of air on the coronary vessels, could not be elicited.

In this case the development of the pneumopericardium was probably slow and insidious. In addition, a definite pneumo-mediastinum was present. We imagine that the course of events was as follows: air entered the mediastinum via the sheaths of the blood- or possibly lymph-vessels, as a result of rupture or leakage from the alveolar walls which had become weakened by the respiratory tract infection. The pneumo-mediastinum so produced may itself have given rise to attacks of dyspnoea and cyanosis. The air in the mediastinum then passed by way of the lymph-clefts to the pericardium, producing a pneumo-pericardium. This was manifested by loud heart sounds, and was also possibly partly responsible for the dyspnoea and cyanosis. As in the majority of such cases, the air was resorbed moderately quickly, so that the most serious results, viz. oedema of the lungs, increased dyspnoea and cyanosis, and finally death, did not occur. (These results can sometimes only be avoided by active intervention, when an outlet for the compressed air must be provided.)

Case 2. A girl, aged five years two months, was transferred on November 9, 1945, to the Children's Clinic from another hospital. A diagnosis of cavernous phthisis had been made, mainly on account of the x-ray appearance of the lungs. A fortnight before admission to the Children's Clinic, the child had complained of pain in the right elbow, and it was found that movement of the arm caused pain in the right shoulder. Three days later the temperature was $38^{\circ}$ to $39 \cdot 4^{\circ} \mathrm{C}$., and though the other joints were not involved and there was no redness or swelling, a diagnosis of rheumatism had been considered. The child had a poor appetite, and had been constipated and drowsy; cough had become more frequent during the five days before transfer, and there was considerable expectoration. The previous history had been essentially negative, and although there had been recurrent cough and poor appetite for the previous six months, there had been no loss of weight. The family was healthy, and since the child's illness both parents had been examined to exclude tuberculosis, with negative result.

ON ADMISSION the child appeared moderately ill, but was active, and her nutritional state was reasonably good. She occasionally coughed up yellowish- green sputum, but no blood. (Repeated examination of the sputum for tubercle bacilli was negative.) There was no cyanosis, clubbing, dyspnoea or adenopathy. The pulse and respiration rates were normal, the temperature $39^{=} \mathrm{C}$. The tongue was furred. The trachea was central. On the right side, in the region of the coracoid process between the sternomastoid and trapezius muscles, it was possible to feel coarse emphysema, which was more obvious after coughing. This area was painful on pressure, as was the right shoulder. There was no redness or swelling of the joints, but passive movement caused pain in the right elbow and shoulder. The percussion note was impaired, anteriorly and posteriorly, over the right upper zone; on auscultation, sub-bronchial respiration could at first be heard with a single rhonchus and increased rocal fremitus. The apex beat was palpable in the fourth

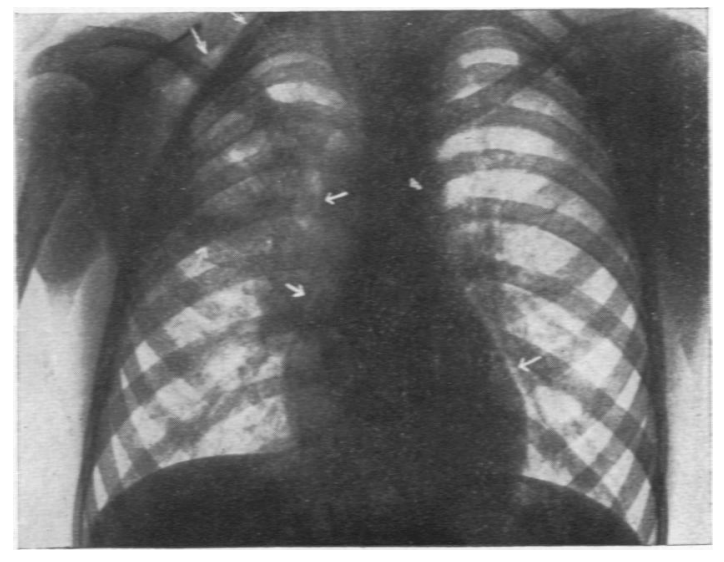

FIG. 3.-X ray of Case 2, taken on Nov. 6.

intercostal space, just inside the nipple line; the area of cardiac dullness was not enlarged; the cardiac sounds were pure, not excessively loud, and regular.

The urine showed a trace of albumin but was otherwise normal. Blood examination showed haemoglobin 59 per cent. (Sahli), red blood cells $3 \cdot 3$ million per c.mm., white blood cells 15,000 per c.mm. (eosinophils 1 per cent., polymorphs 68 per cent., lymphocytes 28 per cent., mononuclears 3 per cent.); toxic granulation was much in evidence.

Radiological eXamination. The $x$ ray taken on November 6 (fig. 3), which was sent to us. showed some characteristic and very large areas of diminished density in the right upper lobe, about $3 \mathrm{~cm}$. in diameter, surrounded by wide borders of increased density. In addition, some shadows indicating subcutaneous emphysema were visible in the right supraclavicular region and over the right side of the thorax. Signs of right-sided mediastinal emphysema were also present, and in the pericardial line a narrow area of translucency was visible (? pneumo-pericardium). The right hilar shadow was enlarged, and calcified foci were present in this area, whilst on the right a linear shadow in the position of the interlobar septum was present.

A subsequent $x$ ray of the chest taken on November 16 (fig. 4) showed that the areas of decreased density in the lung, and also the areas of subcutaneous emphysema, had become smaller: in the right upper iobe, evidence of infiltration was still visible. 


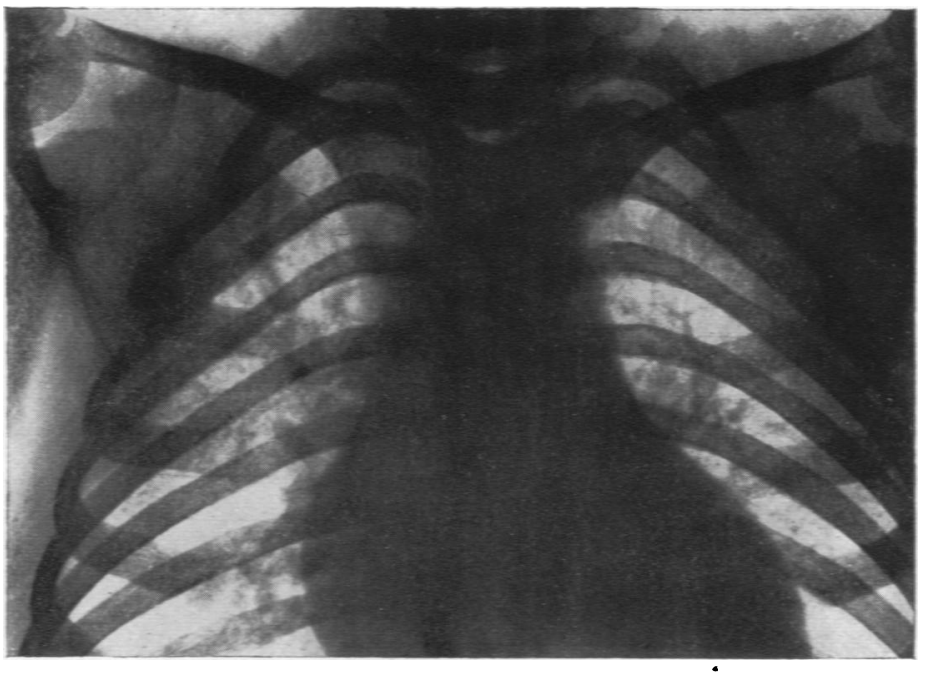

On December 27 radiological examination showed no abnormality apart from the somewhat enlarged right hilar shadow, containing calcified foci, and the linear shadow previously noted in the region of the interlobar septum.

Tuberculin tests. The Pirquet test was negative on admission, but the Mantoux test $(1$ in 1,000$)$ was positive a week later, and again on December 7 . It was concluded that the child had had a previous tuberculous infection, but that her condition on admission was due to localized bullous emphysema complicated by subcutaneous and mediastinal emphysema.

Progress. Following admission, the temperature remained high, and cibazol was prescribed, since in the majority of published cases bullous emphysema has been associated with pneumonia. The temperature then rapidly subsided, and both physical signs and expectoration rapidly decreased. The blood sedimentation rate on November 21 was $24 \mathrm{~mm}$. in one hour, and on December 7 blood examination showed haemoglobin 82 per cent. (Sahli), red blood cells 4.4 million per c.mm., white blood cells 6,500 per c.mm., of which 52 per cent. were polymorphonuclear; toxic granulation was no longer present. Subsequently the child's general condition rapidly improved.

Comment. This second case is an example of the association of symptoms which may follow distension of the alveoli. The occurrence of subcutaneous emphysema of the neck, in the absence of other causes, may be regarded as proof of the presence of mediastinal emphysema, particularly since the initial symptoms (pain in the right shoulder and elbow) have frequently been observed in similar cases. They should be regarded as the result of distension and possibly rupture of tissues through which the air escapes from the mediastinum. (The passage of air from the lung tissue to the mediastinum may also give rise to pains either radiating to the shoulders or down the arms, or localized to the substernal area, which may suggest a false diagnosis of angina pectoris or rheumatism.)

In the first antero-posterior $x$ ray of the chest, we found localized bullous, subcutaneous and mediastinal emphysema present at the same time.
There was also a narrow translucent area along the margin of the pericardium, but (unlike the appearance seen in the first case) we do not feel justified in ascribing this to a pneumo-pericardium. We consider that it is more probably due to the presence of air between the heart and surrounding tissues. In this case, the bullous emphysema which arose as a consequence of a relative stenosis of the bronchus is regarded as primary, and the interstitial emphysema with its various complications as secondary.

Since the child showed a positive Mantoux reaction, the following conditions were considered in differential diagnosis: (1) cavity formation in primary tuberculosis of the lung; (2) localized bullous emphysema resulting from valvular stenosis of a bronchus or bronchioli by tuberculous lesions; and (3) localized bullous emphysema due to valvular stenosis associated with an acute specific infection of the respiratory tract, in a patient who had previously had a tuberculous infection. The first of these diagnoses could be excluded in view of the $\mathrm{x}$-ray appearance of the cavities, their rapid disappearance, and the absence of tubercle bacilli in the sputum. The second diagnosis cannot be excluded in this case. Emphysematous bullae, which during life were regarded as being tuberculous cavities, have most frequently been observed in cases of pulmonary tuberculosis (Miller, 1933; Freedman, 1936; Spivek, 1936), though Benjamin and Childe (1939) have not observed the condition in children with pulmonary tuberculosis without pneumonia. We are, however, inclined to the third diagnosis on account of the rapid disappearance of the cavities, the absence of tubercle bacilli in the sputum, the presence of calcified foci, the rapid subsidence of pyrexia and local signs, and the rapid improvement of the general condition following the administration of cibazol. In both cases, no other treatment was required. Guinea-pig inoculation with fasting stomach-contents gave a negative result. $\mathrm{X}$ ray of the lungs on February 2, 1946 , showed only some small calcified foci at the right hilum.

\section{Summary}

Two cases have been described which clearly 
illustrate the complications, clinical and radiological signs, and symptoms, to which localized bullous and interstitial emphysema may give rise in childhood.

The first patient, an infant, showed a definite pneumo-pericardium occurring as a complication of broncho-pneumonia. Attacks of cyanosis and increased cardiac sounds provided clinical confirmation of the diagnosis.

The second patient, a girl aged five years, showed symptoms and signs of bullous and subcutaneous emphysema occurring simultaneously, with, in addition, radiological evidence of mediastinal emphysema and air situated around the heart. Here the localized bullous emphysema must have preceded the occurrence of the interstitial emphysema. Attention is drawn to the particular clinical signs which were present in this case.

\section{REFERENCES}

Benjamin, B., and Childe, A. E. (1939). J. Pediat., 15 620.

Caffey, J. (1940). Amer. J. Dis. Child., 60, 586.

Creveld, S. van, and Poorten, F. H. ter (1935). Arch. Dis. Childh., 10, 125.

Fischer, J. H., and Macklin, C. C. (1940). Amer. J. Dis. Child., 60, 102.

Freedman, E. (1936). Amer. J. Roentgenol., 35, 324.

Graebner, H.(1939). Arch. Otolaryng. Chicago, 29, 446.

Hamman, L. (1945). J. Amer. med. Ass., 128, 1.

Leopold, J. S., and Kratzman, E. A. (1945). Amer. J. Dis. Child., 69, 287.

Macklin, C. C. (1939). Arch. intern. Med., 64, 913.

- (1937). Canad. Med. J., 36, 414.

Miller, W. S. (1933). Amer. Rev. Tuberc., 28, 359.

Spivek, M. L. (1936). Amer. J. Dis. Child., 51, 69.

Tillema, E. A. (1940). Ned. T̈̈dschr. Geneesk., 84, 3666.

Wissler, H. (1938). Arch. Kinderheilk., 113, 16. 